\title{
Measurement of Functional Residual Capacity in Rabbits and Children Using an Ultrasonic Flow Meter
}

\author{
ANDREAS SCHIBLER AND ROBERT HENNING
}

Paediatric Intensive Care Unit, Royal Children's Hospital Melbourne, Parkville 3052, Victoria, Australia.

\begin{tabular}{|c|c|}
\hline \multicolumn{2}{|c|}{ ABSTRACT } \\
\hline 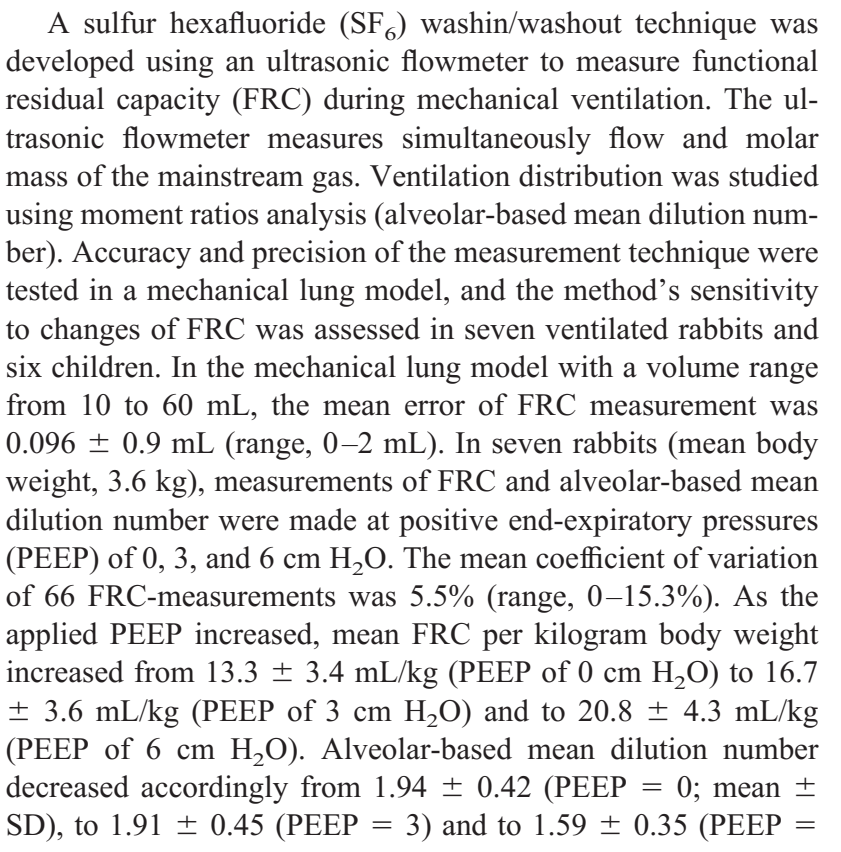 & 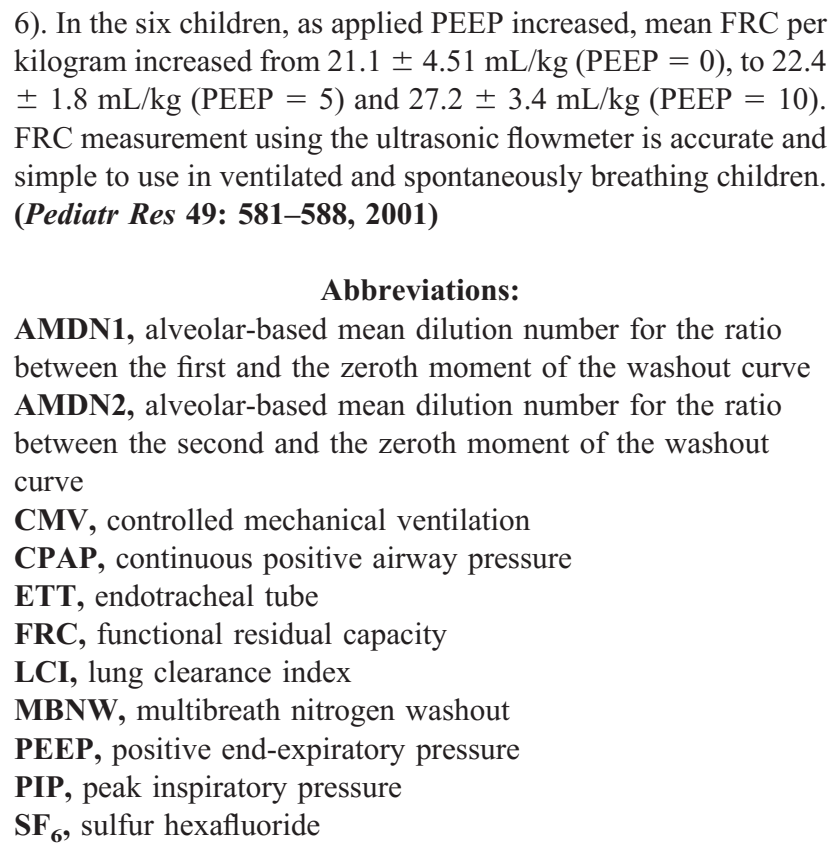 \\
\hline
\end{tabular}

The monitoring of FRC is an important tool for interpreting volume-dependent pulmonary mechanics (e.g. lung elastic recoil pressure or airway resistance). Since the open-circuit MBNW was first described by Darling et al. in 1940 (1), several investigators have used gas washout techniques to measure FRC in spontaneously breathing $(2,3)$ and mechanically ventilated patients $(4-6)$. Other gas washout techniques involve helium dilution (7), argon washout (8), or washout of $\mathrm{SF}_{6}(9)$. Each of these techniques has its own limitations. The MBNW can only be accurately performed if the patient's oxygen requirement does not exceed a certain amount (4-6).

Received January 13, 2000; accepted July 18, 2000.

Correspondence and reprint requests: Andreas Schibler, M.D., Children's Hospital of the University of Bern, Pediatric Intensive Care Unit, 3010 Inselspital Bern, Switzerland; e-mail: andreas.schibler@insel.ch

Supported by a grant from the Swiss Cystic Fibrosis Foundation and the Novartis Foundation.
Although MBNW can be performed easily, the considerable changes in gas viscosity during the washout maneuver significantly affect the accuracy of the gas flow measurement by pneumotachography $(10,11)$. The helium dilution technique is based on a closed circuit, and a leak in the measuring system reduces its accuracy (7). $\mathrm{SF}_{6}$ washout is a potentially suitable technique for children, including those who require near $100 \%$ oxygen to maintain normal $\mathrm{Hb}$ saturation. There are a few studies using $\mathrm{SF}_{6}$ as a washout gas (12-14) and a mass spectrometer or an infrared analyzer to measure $\mathrm{SF}_{6}$ concentration. Neither technique is commercially available for routine measurement. The infrared analyzer has a low signal-to-noise ratio and a response time of $20 \mathrm{~ms}$ or more. Airflow in these studies was usually measured with a pneumotachograph, and in most cases allowance was not made for changes in gas density. In this study, we investigated a $\mathrm{SF}_{6}$ washin/washout technique using a mainstream ultrasonic flowmeter. With this technique, 
flow and gas density of the inspiratory and expiratory gas can be assessed simultaneously. The concentration of a tracer gas during washin or washout can be obtained by measuring gas density. This allows calculation of FRC and assessment of distribution homogeneity (using moment ratios). The aim of this study was to assess the accuracy and reproducibility of such a technique in a mechanical lung model and in spontaneously breathing and mechanically ventilated rabbits, as well as in patients being ventilated for respiratory distress.

\section{METHODS}

The FRC measurement unit (Fig. 1) consists of a ventilator with a constant-circuit flow, a system that delivers $\mathrm{SF}_{6}$ into the ventilator circuit, an ultrasonic flowmeter placed between the ventilator circuit and the ETT, and a personal computer for triggering the $\mathrm{SF}_{6}$ delivery system and for data acquisition.

Ventilator system. For measurements in the mechanical lung model and in the animal experiment, a Bourns Bear Cub infant ventilator (Palm Springs, CA, U.S.A) with a constant-circuit flow was used. The ventilator provided conventional modes of CMV and CPAP. PIP and PEEP were controlled by the ventilator's pressure valves. During animal studies inspiratory gas was humidified and heated to $34.5^{\circ} \mathrm{C}$ (respiratory humidifier MR700, Fisher \& Paykel). The children in the study were ventilated with a Bird V.I.P. ventilator, which delivers a constant-circuit flow. Synchronized intermittent mandatory ventilation was used in the human study. The inspired gas was heated to $37^{\circ} \mathrm{C}$ and was $100 \%$ humidified. Airway pressure was measured using the analog output of the pressure signal of a Florian Neonatal Respiratory Monitor. The pressure signal was calibrated with a two-point calibration using a water column. The analog signal was fed into the Spiroson control unit.

Ultrasonic flowmeter. In its basic version, the equipment (Spiroson Scientific, Eco Medics AG, Dürnten, Switzerland) consists of a control unit, an external power supply, and the ultrasonic flowmeter. The ultrasonic flowmeter is operated by a personal computer using dedicated software. The design of

pressure signal

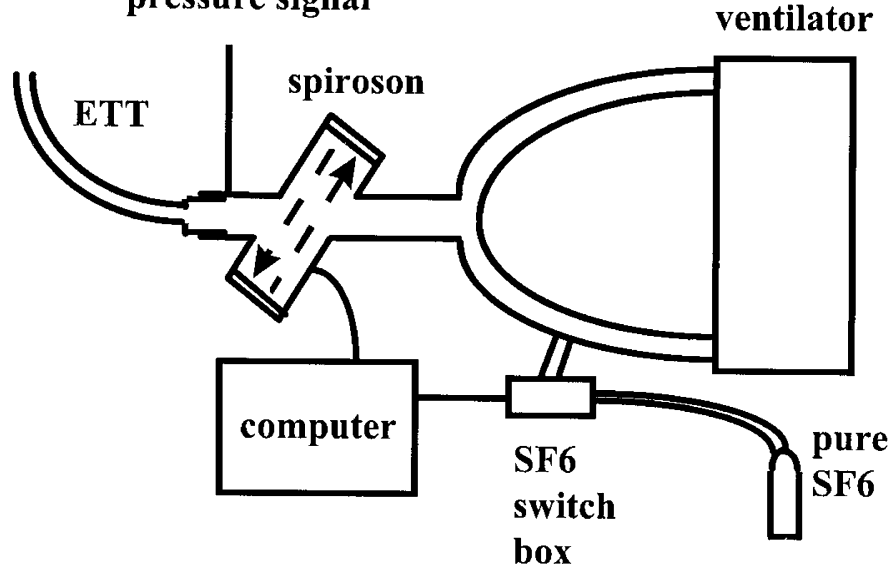

Fig. 1. The $\mathrm{SF}_{6}$ and flow measuring unit. The all-in-one sensor (Spiroson) measures flow, volume, and molar mass of the respiratory gas. $\mathrm{SF}_{6}$ is added into the circuit over a delivering system. the flowmeter is shown in Figure 1 and is based on the principle that sound traveling through a streaming medium is accelerated or slowed by the movement of the medium, causing, for a fixed distance across the medium, a decrease in the downstream and an increase in the upstream transit times. The changes in transit time can be related to the flow velocity (15). Two ultrasonic transducers are mounted in recessed wells on opposite sides of the tube at an angle of $40^{\circ}$ to the flow axis. Both transducers emit ultrasonic pulses of $50 \mathrm{kHz}$, which pass in opposite directions through the moving medium. Two different sizes of flowmeter were used. The smaller had a dead space of $1.3 \mathrm{~mL}$ and cross-sectional internal diameter of $5 \mathrm{~mm}$ (resistance of $1.1 \mathrm{kPa} / \mathrm{L} / \mathrm{s}$ ), and the larger a dead space of 3.8 $\mathrm{mL}$ and a diameter of $12 \mathrm{~mm}$ (resistance of $0.6 \mathrm{kPa} / \mathrm{L} / \mathrm{s}$ ). Accuracy of both flowmeters was assessed with a Calibration Analyzer (series RT-200, Timeter Instrument Corporation, BC Group International Inc, St. Louis, MO, U.S.A.). Daily calibration of the ultrasonic flowmeter was performed with a high-precision 100-mL calibration syringe (Hans Rudolph, 5510 Series). The ultrasonic flowmeter was considered to be calibrated adequately if integrated volume was within $1 \%$ of $100 \mathrm{~mL}$.

Molar mass. Molar mass (grams per mole) can be computed from transit times and temperature along the sound transmission path. The transit time can be measured with high accuracy, and its value is directly proportional to the density of the medium. Hence, using the formula

$$
\varphi=(M \cdot p) /(R \cdot T)
$$

the molar mass can be calculated (where $\phi$ is the gas density, $M$ is the molar mass, $p$ is the pressure, $R$ is the gas constant, and $T$ is the temperature). However, the precise temperature along the sound transmission path must be determined. Because of the complexity of the determination of the temperature along the sound transmission path, native molar mass was introduced. Native molar mass is computed by assuming a constant, predefined temperature along the sound transmission path. Native molar mass is therefore a standardized form of the molar mass. By measuring temperature inside the sound path, the native molar mass signal can be converted into the (final) molar mass signal used for gas analysis. If minor changes in temperature are present (using heated and humidified inspiratory gas), the changes of temperature along the sound path can be simulated using a temperature model. The temperature model basically uses the measured flow, instrumental dead space, and the preset temperature of the inspiratory and expiratory gas to calculate the temperature changes along the sound transmission path. Pressure changes during CMV also alter transit times of the ultrasonic pulse. During high pressure, the transit time is shorter than during low pressure, leading to underestimation of molar mass. Figure 2 shows the relationship between pressure and the molar mass signal. For this reason, the ultrasonic flowmeter was filled with either room air or with a gas mixture containing room air and $6 \% \mathrm{SF}_{6}$. The flowmeter was then pressurised from 0 to $40 \mathrm{~cm} \mathrm{H}_{2} \mathrm{O}$, and the molar mass signal was measured. Figure 3 shows flow, volume, pressure, and molar mass during mechanical ventilation of a rabbit using 


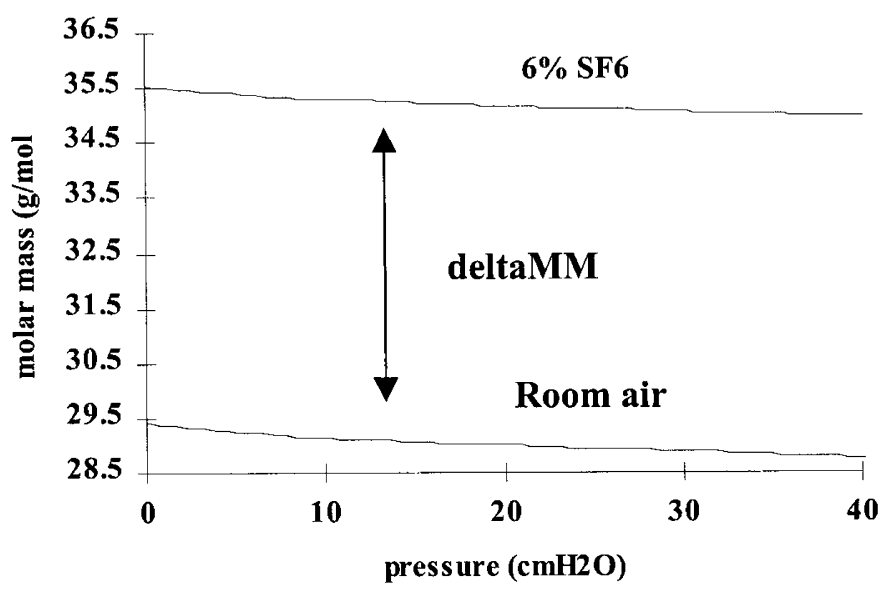

Fig. 2. The relationship between pressure and molar mass signal. The lower curve shows the response of the molar mass signal to increased pressure inside the ultrasonic flowmeter when room air is used. The upper curve shows the same response of the molar mass signal to increased pressure when the gas inside the ultrasonic flowmeter contains a mixture of $94 \%$ room air and $6 \%$ $\mathrm{SF}_{6}$. Note that both curves show a decrease of molar mass signal with higher pressure, but the difference between the curves (deltaMM) is constant.
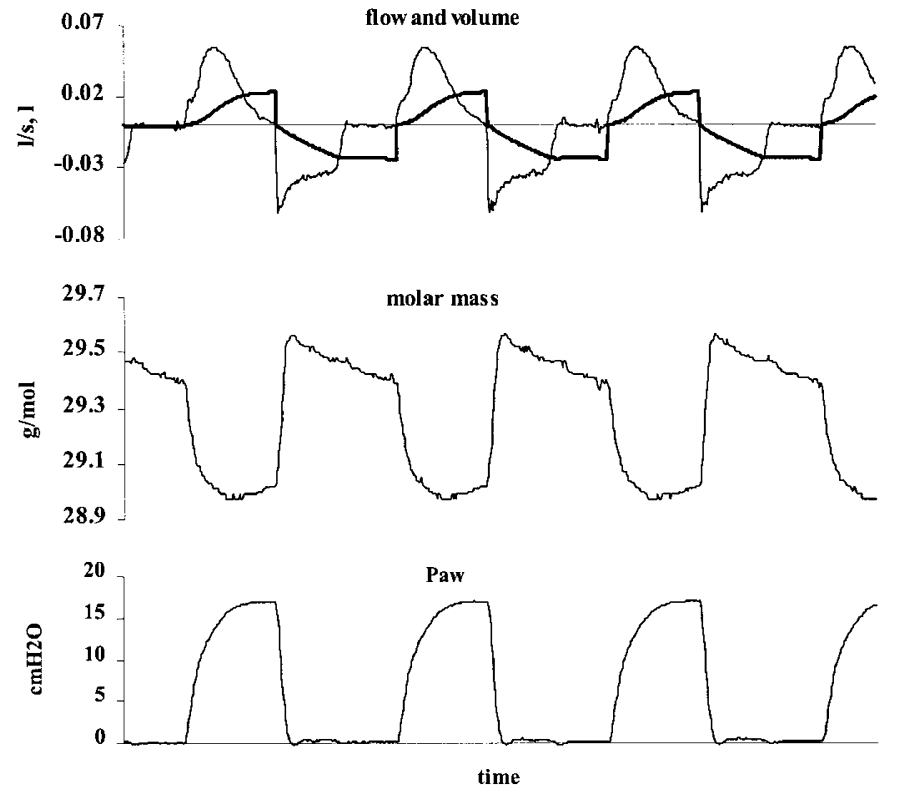

Fig. 3. Flow, volume, pressure, and molar mass signal. The three panels show flow (thin line) and volume (bold line) changes as well as changes in the molar mass signal and airway pressure (Paw) during CMV. The changes in molar mass reflect changes in pressure, temperature, and $\mathrm{CO}_{2}$ removal during expiration.

PIP of $16 \mathrm{~cm} \mathrm{H}_{2} \mathrm{O}$ and PEEP of $0 \mathrm{~cm} \mathrm{H}_{2} \mathrm{O}$. The composition of the inspiratory gas differs from the expiratory because of pulmonary gas exchange. Normally the inspiratory gas consists of $21 \%$ oxygen and $79 \%$ nitrogen, resulting in a molar mass of $28.85 \mathrm{~g} / \mathrm{mol}$. During expiration, the molar mass is slightly higher because of the presence of $\mathrm{CO}_{2}$, the molar mass of which is approximately $29.09 \mathrm{~g} / \mathrm{mol}$. To take account of these effects of temperature, airway pressure, and gas composition on the molar mass signal, we subtracted from the measured molar mass during washin and washout the baseline molar mass, which was obtained during the corresponding phase of tidal breathing before the $\mathrm{SF}_{6}$ washin (assuming that during a measurement the breathing conditions remain constant). The difference between measured and baseline molar mass is called delta MM. The washin or washout curve of $\mathrm{SF}_{6}$ was described using deltaMM. To obtain the instantaneous $\mathrm{SF}_{6}$ fraction at every moment during washin and washout, deltaMM was divided by the molar mass of $\mathrm{SF}_{6}(146.05 \mathrm{~g} / \mathrm{mol})$. The molar mass can be assessed with the Spiroson ultrasonic flowmeter over the range of molar mass of 20 to $45 \mathrm{~g} / \mathrm{mol}$ with a precision of $0.01 \mathrm{~g} / \mathrm{mol}$. A two-point calibration of the molar mass signal was performed using room air and $6 \% \mathrm{SF}_{6}$.

$S F_{6}$ delivery system. A low, constant $\mathrm{SF}_{6}$ flow was delivered by a high-pressure $\mathrm{SF}_{6}$ cylinder with an adjustable regulator and with a low-range flowmeter. The relationship between $\mathrm{SF}_{6}$ flow and circuit flow was 1:20. A pressure-driven blender controlled by the computer was used to switch the $\mathrm{SF}_{6}$ supply on and off. During the first phase of a washin process, the lungs of the patient were filled with $\mathrm{SF}_{6}$ until $<0.05 \%$ change in molar mass at end expiration and the next could be observed. Switching off the $\mathrm{SF}_{6}$ supply at the beginning of the next expiration then started the washout of the $\mathrm{SF}_{6}$. The purity of $\mathrm{SF}_{6}$ with $99.99 \%$ was guaranteed by the manufacturer (Air Liquide Healthcare, Melbourne, Australia). However, it is still possible that the remaining $0.01 \%$ contains toxic contaminants (such as $\mathrm{SF}_{5}$ ): the concentration of these contaminants would be $<0.0025 \%$ when we use a $\mathrm{SF}_{6}$ concentration of 2 to $4 \%$.

Data acquisition. The signal of flow, molar mass, and the analog signal of pressure were acquired with the Spiroson control unit, converted to digital signals, and sampled at a rate of $100 \mathrm{~Hz}$. For data acquisition and signal calibration (flow, temperature, pressure), the software provided with the ultrasonic flowmeter was used. Clicking on a switch icon on the computer screen started $\mathrm{SF}_{6}$ washin. After six tidal breaths, the computer postponed the final on-switch output to the $\mathrm{SF}_{6}$ valve to the start of the next expiration. Thus, the tracer gas primed the inspiratory limb of the ventilator circuit during that expiration and was delivered at a constant concentration to the subject during the next inspiration. Flow, volume, pressure, and molar mass were displayed on the computer screen. Once equilibrium of end-tidal molar mass was achieved, the $\mathrm{SF}_{6}$ valve was switched off and the ventilator circuit was flushed with fresh gas free of $\mathrm{SF}_{6}$ during the next expiration. The same fresh gas was inspired during the washout. Data acquisition was stopped when no further change in end-tidal molar mass could be observed. All signals were stored and processed on a computer hard disk. Figure 4 shows a typical $\mathrm{SF}_{6}$ washin and washout during $\mathrm{CMV}$ using native molar mass.

Data analysis. For calculation of FRC and analysis of ventilation distribution homogeneity, software written by one of the authors (A.S.) in LabView 5.0 (National Instruments, Austin, TX, U.S.A.) was used. Flow and volume were corrected to BTPS. The molar mass signal was calculated using the temperature simulation mentioned earlier. From the molar mass, deltaMM and $\mathrm{SF}_{6}$ fraction were obtained, and the $\mathrm{SF}_{6}$ flow was calculated as the product of instantaneous airflow and instantaneous $\mathrm{SF}_{6}$ concentration. The $\mathrm{SF}_{6}$ flow was integrated over time to give the expired $\mathrm{SF}_{6}$ volume. The FRC of washout was obtained by dividing the measured expired $\mathrm{SF}_{6}$ volume 


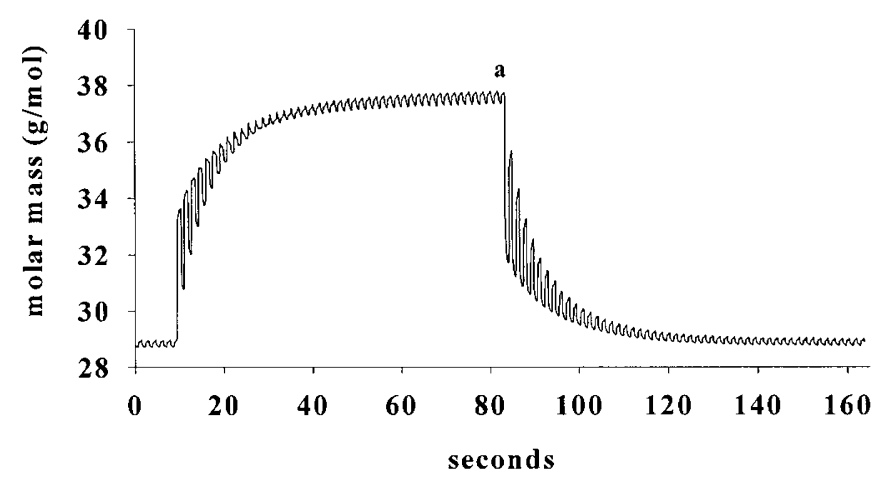

Fig. 4. Typical washin and washout of $\mathrm{SF}_{6}$. Washout of $\mathrm{SF}_{6}$ is initiated when end-expiratory $\mathrm{SF}_{6}$ concentration is constant (point $a$ ).

with the end-tidal $\mathrm{SF}_{6}$ concentration before washout. The initial $\mathrm{SF}_{6}$ volume within the flowmeter and the connectors was subtracted from the total of the exhaled $\mathrm{SF}_{6}$ volume. The FRC of the washin was determined similarly using the mathematical mirror image of the washout signal. FRC was measured in duplicate for each washin and washout. The FRC calculation does not include the $\mathrm{SF}_{6}$ remaining in the lung when washout is terminated, which introduces a small error.

To assess ventilation distribution homogeneity, moment ratio analysis of the $\mathrm{SF}_{6}$ washout curve was performed. In the present study we used the alveolar-based mean dilution number to describe gas-mixing characteristics $(16,17)$. The moment ratios were calculated with the cumulated expired alveolar volume, normalized with $\mathrm{FRC}$, and $\mathrm{SF}_{6}$ concentration as variables. The calculation of the moment ratios started with the first breath immediately after start of washin or washout and was stopped after 10 turnovers. Dead space was measured during the first three exhalations during washin and washout and was calculated with the Bohr formula (16). Quantitatively, the moments can be understood as follows: the zeroth moment $\left(\mathrm{m}_{0}\right)$ is simply the area under the $\mathrm{SF}_{6}$ washout curve ( $y$ axis) using the dilution number (cumulative expired alveolar volume divided by FRC) as $x$ axis, while the first and second moments $\left(\mathrm{m}_{1}, \mathrm{~m}_{2}\right)$ and higher moments are weighted values of area segments under the washout curve. The tail regions of the curve are given more weight for successively higher moments. AMDN1 is the ratio between $m_{1}$ and $m_{0}$, and AMDN2 is the ratio between $m_{2}$ and $m_{0}$. In addition to moment ratios, we calculated the LCI (17). The LCI is the number of turnovers (cumulative expired volume/FRC) required to reduce the endtidal tracer gas concentration to one fortieth of the concentration at the start of washout.

Mechanical lung model. A previously described lung model (12) was used to assess the accuracy of the $\mathrm{SF}_{6}$ washin and washout. The model consisted of a thin-walled balloon attached on top of a syringe. The balloon and the top of the syringe were placed inside a glass bottle, which was slightly pressurized to guarantee that the balloon was completely empty at end expiration. The model was ventilated through an endotracheal tube, which was inserted through the plunger of the syringe. The FRC of the lung model was the volume inside the syringe and the dead space of the connecting ETT. FRC could be varied between 10 and $60 \mathrm{~mL}$ by sliding the syringe's plunger up and down. The lung model was ventilated with two different tidal volumes (20 and $30 \mathrm{~mL})$ and inspiratory pressures (20 and $40 \mathrm{~cm} \mathrm{H}_{2} \mathrm{O}$ ).

Animal study. The Animal Ethics Committee of the Royal Children's Hospital, Melbourne, Australia, approved the animal study. Seven adult New Zealand White rabbits (mean body weight, $3.6 \mathrm{~kg}$; range, $2.67-4.3 \mathrm{~kg}$ ) were anesthetized with thiopentone $20-25 \mathrm{mg} / \mathrm{kg}$ i.v., followed by infusion of $20-25$ $\mathrm{mg} / \mathrm{kg} / \mathrm{h}$ i.v. All the animals were intubated with a cuffed ETT (Malinckrodt, size $3.5 \mathrm{~mm}$ ). The anesthetic level of the animals was maintained at surgical anesthesia to minimize depression of spontaneous breathing. A Bourns Bear Cub infant ventilator was used to apply CPAP and CMV. An i.v. infusion of Hartmann's solution $4 \mathrm{~mL} / \mathrm{kg} / \mathrm{h}$ was maintained throughout the study. ECG, invasive blood pressure, and transcutaneous oxygen saturation were monitored continuously. Each animal was exposed during spontaneous breathing to three different levels of CPAP of 0,3 , and $6 \mathrm{~cm} \mathrm{H}_{2} \mathrm{O}$. At least 5 min was allowed for stabilization at each level before measurements were made with the small ultrasonic flowmeter. $\mathrm{SF}_{6}$ washin and washout were repeated at least three times at each level of CPAP, resulting in six FRC values per level. CMV was then started without neuromuscular blockade to allow spontaneous breathing during CMV. All animals were ventilated with a PIP of 14 to $16 \mathrm{~cm} \mathrm{H}_{2} \mathrm{O}$ at a rate of $38 / \mathrm{min}$ and an inspiratory time of $0.8 \mathrm{~s}$. Inspired fraction of oxygen was set at 30\%. PEEP level was elevated stepwise from 0 to 3 and to $6 \mathrm{~cm} \mathrm{H}_{2} \mathrm{O}$. At each level three $\mathrm{SF}_{6}$ washin and washout measurements were performed.

To compare the $\mathrm{SF}_{6}$ washin and washout technique to a near gold standard, we used a technique that has been described by Sivan et al. (18). Accordingly we inflated the lungs of the animals at end expiration by clamping the ETT followed by inflating the lungs with $20 \mathrm{~mL}$ of the ventilatory gas. The ventilator circuit was then primed with the $\mathrm{SF}_{6}$ mixture. The clamped ETT was reopened at the beginning of an inspiration and $\mathrm{SF}_{6}$ washin was performed. The FRC measured after 20 $\mathrm{mL}$ inflation was compared with baseline measurements without lung inflation. A total of six inflation tests were performed per animal.

Human study. The Human Ethics Committee of the Royal Children's Hospital, Melbourne, Australia, approved the human study. Six children and infants (two girls and four boys) who required mechanical ventilation were measured on the intensive care unit of the Royal Children's Hospital Melbourne. The ages of the children ranged from 5 mo to $12 \mathrm{y}$, with a body weight from 5 to $28 \mathrm{~kg}$. Three patients suffered from pneumonia, one patient from interstitial lung disease, one patient had suffered from near-drowning, and one patient had had cardiac surgery (atrial septal defect repair). To ensure that a leak around the ETT did not invalidate the measurements, these were made only when inspired and expired tidal volumes were nearly equal $(<1 \%$ difference). The large ultrasonic flowmeter was used for children weighing $>12 \mathrm{~kg}$, and the small flowmeter, in those weighing $\leq 12 \mathrm{~kg}$. All children were ventilated using a synchronized intermittent mandatory ventilation mode that allowed spontaneous breathing. Each patient was exposed to PEEP levels of 0,5 , and $10 \mathrm{~cm} \mathrm{H}_{2} \mathrm{O} . \mathrm{SF}_{6}$ 
washin and washout were measured in triplicate on each level. Measurements were made at each PEEP level after a waiting time of $30 \mathrm{~min}$.

Statistics. Values are presented as mean \pm SD. Reproducibility of the system in vitro and in vivo was evaluated by calculating the coefficient of variation. The accuracy of the measurements (limits of agreement) in the mechanical lung model and of the inflation results in the animal study was assessed with the difference between the measured volume and the mean of measured and preset volumes (19). To examine the effect of tidal volume and PIP on accuracy of FRC measurements in the lung model, a two-way ANOVA was used. Percent error was defined as $100 \times$ absolute value of (preset volume - measured volume)/preset volume. Because of small sample numbers, a Wilcoxon signed rank test was used to compare paired FRC values and moment ratios at different CPAP and PEEP levels. A $p$ value of $<0.05$ was considered as significant.

\section{RESULTS}

Accuracy of the ultrasonic flow head. Both flow heads showed an accuracy within $3 \%$ (flow range, $\pm 0.006-1 \mathrm{~L} / \mathrm{s}$ for the small, and $0.006-3 \mathrm{~L} / \mathrm{s}$ for the larger flow head). Humidity, varying airway pressure during $\mathrm{CMV}$, and changes of gas composition because of $\mathrm{SF}_{6}$ mixture caused an error of $<3 \%$ in volume measurement. The calibration of the molar mass with increasing $\mathrm{SF}_{6}$ concentration was tested using five different $\mathrm{SF}_{6}$ mixtures $(2,4,6,8$, and $10 \%)$ and has been proven to be linear (error from line of identity $<1 \%$ ). Applying different pressures as shown in Figure 2 caused a baseline shift in the molar mass signal but had no effect on the linearity itself of the $\mathrm{SF}_{6}$ signal (error $<1.1 \%$ ).

Limits of agreement. The mean error of FRC volume measurement in the mechanical lung model was $0.096 \pm 0.9 \mathrm{~mL}$ (range, $0-2 \mathrm{~mL}$ ) or, expressed in percentage, $0.59 \pm 5.3 \%$ (range, $0-9.3 \%$ ). Figure 5 shows the limits of agreement using

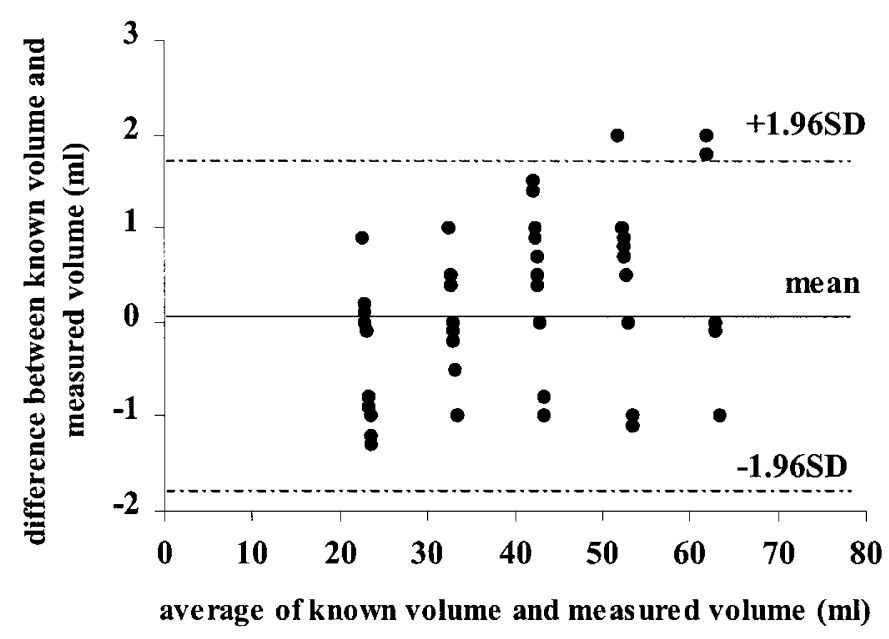

Fig. 5. Limits of agreement. The average of the measured and known FRC of a mechanical lung model is plotted against the differences. The horizontal lines indicate the $95 \%$ confidence interval $(1.96 \times \mathrm{SD}$ of the difference between known and measured volumes). The mean volume error was $0.096 \pm$ $0.9 \mathrm{~mL}$. a Bland and Altman plot (19). Change in tidal volume and PIP did not affect the accuracy of FRC measurement in the lung model $(p<0.001)$. FRC measurement at six preset volumes was highly reproducible at different tidal volumes and pressures (coefficient of variation, 2.3-7.1\%; mean, 4.3\%).

Animal study. The results of the FRC measurements at each CPAP and PEEP level are shown in Table 1. Each FRC value is the average of six measurements. Each increase of CPAP (during spontaneous breathing) and PEEP (during CMV) caused a significant stepwise increase in FRC in every animal $(p<0.001)$. This increase in volume was far greater than the variation of the method itself. The average coefficient of variation during spontaneous breathing (66 FRC measurements) at different CPAP levels was 7.1\% (range, 0.6-15.6\%) and during CMV, 5.5\% (range, $0-15.3 \%$ ). FRC measured during washin showed no significant difference from FRC measured during washout. The average washin FRC during spontaneous breathing on CPAP was $50.79 \mathrm{~mL}$ and average washout FRC was $49.82 \mathrm{~mL}(p=0.25)$. During CMV, the average washin FRC was $57.35 \mathrm{~mL}$ and washout FRC was $57.20 \mathrm{~mL}(p=0.4)$.

The values of the moment ratios AMDN1 and AMDN2 are shown in Table 1. These decreased as the level of CPAP or PEEP increased, indicating an improvement in the distribution of ventilation. Not all changes reached significance as shown in Table 1. Both AMDN1 and AMDN2 were significantly lower at each CPAP level during spontaneous breathing than the corresponding moment ratios during CMV. Accordingly, the LCI decreased with increasing CPAP or PEEP level.

Lung inflation test. The mean baseline measured FRC value (total 42 measurements) before the lung inflation was 36.91 $\mathrm{mL}$ and after $20 \mathrm{~mL}$ inflation, $57.06 \mathrm{~mL}$, resulting in an average measured inflation of $20.15 \pm 1.5 \mathrm{~mL}$. Mean error of the measurement compared with a true inflation volume of 20 $\mathrm{mL}$ was $4.3 \%$ (range, $0-6.65 \%$ ).

Human study. The results of the FRC measurements obtained at different PEEP levels are shown in Table 2. Each FRC value is the average of six measurements. The mean coefficient of variation of the FRC measurements was $8.0 \%$ (range, 2.84-15.1\%). As expected, the FRC increased with increasing PEEP level. Increasing the PEEP from 0 to $10 \mathrm{~cm} \mathrm{H}_{2} \mathrm{O}$ significantly increased the FRC, but the change from 0 to $5 \mathrm{~cm}$ $\mathrm{H}_{2} \mathrm{O}$ did not. LCI, AMDN1, and AMND2 fell as PEEP increased, but the changes did not reach a significant level.

\section{DISCUSSION}

In this study, a new approach to measurement of FRC using $\mathrm{SF}_{6}$ washin and washout technique has been evaluated. Instead of a tracer-specific analyzer such as a mass spectrometer, nitrogen analyzer, or infrared $\mathrm{SF}_{6}$ analyzer, we used the molar mass of the ventilation gas to estimate $\mathrm{SF}_{6}$ concentration. The high molar mass of $\mathrm{SF}_{6}(146 \mathrm{~g} / \mathrm{mol})$ gives an excellent signal response when an ultrasonic flowmeter is used to measure gas density. The present $\mathrm{SF}_{6}$ washin and washout technique can be used during spontaneous breathing as well as during CMV without interfering with patient care. In a mechanical lung model, we could demonstrate the high accuracy of the measurement technique (mean of the absolute percent error was 
Table 1. FRC values and moment ratios of the $S F_{6}$ washin and washout in seven intubated rabbits

\begin{tabular}{|c|c|c|c|c|c|}
\hline Ventilation node & $\begin{array}{c}\text { FRC } \\
(\mathrm{mL} / \mathrm{kg})\end{array}$ & $\begin{array}{l}\mathrm{CV} \\
(\%)\end{array}$ & LCI & AMDN1 & AMDN2 \\
\hline \multicolumn{6}{|l|}{$\mathbf{C P A P}\left(\mathrm{cm} \mathrm{H}_{2} \mathrm{O}\right)$} \\
\hline 0 & $9.2 \pm 1.9^{*}$ & 10.5 & $7.7 \pm 2.5 \dagger$ & $1.94 \pm 0.42$ & $6.73 \pm 4.01$ \\
\hline 6 & $19.6 \pm 4.0^{*}$ & 5.5 & $6.3 \pm 1.8 \dagger$ & $1.59 \pm 0.35 \ddagger$ & $4.69 \pm 2.31 \%$ \\
\hline \multicolumn{6}{|l|}{$\operatorname{PEEP}\left(\mathrm{cm} \mathrm{H}_{2} \mathrm{O}\right)$} \\
\hline 0 & $13.3 \pm 3.4^{*}$ & 8.2 & $8.3 \pm 2.67 \dagger$ & $2.46 \pm 0.48 \S$ & $9.53 \pm 4.18$ \\
\hline
\end{tabular}

Results are shown as mean $\pm \mathrm{SD}$, except for $\mathrm{CV}$. Abbreviation: $\mathrm{CV}$, coefficient of variation.

* Every increase in CPAP or PEEP from 0 to 3 and from 3 to $6 \mathrm{~cm} \mathrm{H}_{2} \mathrm{O}$ caused a significant increase in FRC $(p<0.001)$

$\dagger$ LCI was significantly lower at CPAP or PEEP of 6 compared with $0 \mathrm{~cm} \mathrm{H}_{2} \mathrm{O}(p<0.05)$.

$\ddagger$ Ventilation distribution described by AMDN1 and AMDN2 during spontaneous breathing improved significantly at $\mathrm{CPAP}$ of $6 \mathrm{~cm} \mathrm{H}_{2} \mathrm{O}$ compared with 0 or $3 \mathrm{~cm} \mathrm{H} \mathrm{H}_{2} \mathrm{O}(p<0.05)$.

$\S$ During CMV every increase of PEEP caused a significant decrease of AMDN1 $(p<0.05)$.

IIADN2 was only significantly lower at PEEP of 6 compared with 0 and $3 \mathrm{~cm} \mathrm{H}_{2} \mathrm{O}(p<0.05)$. No significant difference of AMDN2 was found between PEEP of 0 and $3 \mathrm{~cm} \mathrm{H}_{2} \mathrm{O}$. AMDN1 and AMDN2 measured during spontaneous breathing were significantly $(p<0.05)$ lower than the corresponding values measured during CMV.

Table 2. FRC values and moment ratios of the $S F_{6}$ washin and washout in six intubated children

\begin{tabular}{|c|c|c|c|c|c|}
\hline $\begin{array}{c}\text { PEEP } \\
\left(\mathrm{cm} \mathrm{H} \mathrm{H}_{2} \mathrm{O}\right)\end{array}$ & $\begin{array}{c}\text { FRC } \\
(\mathrm{mL} / \mathrm{kg})\end{array}$ & $\begin{array}{l}\mathrm{CV} \\
(\%) \\
\end{array}$ & LCI & AMDN1 & AMDN2 \\
\hline 0 & $21.1 \pm 4.51$ & 9.1 & $6.80 \pm 0.60$ & $1.88 \pm 0.16$ & $5.75 \pm 0.94$ \\
\hline 5 & $22.4 \pm 1.8$ & 6.2 & $6.15 \pm 0.44$ & $1.69 \pm 0.17$ & $4.46 \pm 0.81$ \\
\hline
\end{tabular}

Results are presented as mean $\pm \mathrm{SD}$, except for CV. Abbreviation: CV, coefficient of variation.

* Measured FRC increased significantly at $10 \mathrm{~cm} \mathrm{H}_{2} \mathrm{O}$ of PEEP compared with 5 or $0(p<0.05)$. LCI, AMDN1, and AMDN2 decreased as PEEP increased, but the difference did not reach significance.

$0.59 \pm 5.3 \%$ ) and good reproducibility (mean coefficient of variation, $4.3 \%$ ). Bench tests showed similar accuracy and reproducibility for the open-circuit nitrogen washout technique (5). There is no gold standard technique for FRC measurement in vivo because every technique has its own limitations. Sivan et al. (18) have shown that FRC measurement using the nitrogen washout method measures accurately an artificial inflation of the lungs and concluded that nitrogen washout measures true FRC. Using the same artificial inflation technique of the lungs, we demonstrated that a preset volume increment could be measured with high accuracy using the molar mass method. However, the $\mathrm{SF}_{6}$ washin and washout technique in this study needs to be compared in future with standard lung volume measurements such as whole body plethysmography or nitrogen washout.

Some specific features of the ultrasonic flowmeter make it highly suitable for breath-by-breath analysis of a gas washout. The ultrasonic flowmeter estimates flow and molar mass by measuring changes in transit time over a fixed distance inside the flowmeter. Both signals are measured simultaneously, and no delay time correction is necessary. This is an important advantage, as several studies $(6,20)$ have shown that when gas fractions are measured by sidestream sampling, there is a substantial delay between gas analysis signal and the mainstream gas flow measurement signal, mainly caused by the transport time of the sampled gas. Furthermore, flow measurement with a conventional pneumotachograph must be corrected for changes in gas viscosity during gas washout (21), requiring cumbersome calibration. If no accurate correction for changes in gas viscosity is performed during nitrogen washout, an error in volume measurement as high as $12 \%$ can occur (22). Since the introduction of computer analysis, these disadvantages are less important, but changes in measurement conditions always require recalibration. Although the gas composition does affect the speed of sound, from a theoretical point of view this has negligible consequences for the ultrasonic flowmeter (15). As long as the flow velocity is much lower than the speed of sound, the estimated error caused by variation in gas mixture cannot be $>3 \%$. Flow is obtained by measuring the transit times of the $80-\mathrm{kHz}$ ultrasonic sound across the flow channel in opposite directions $\left(t_{\mathrm{u}}\right.$ is the upstream transit time and $t_{\mathrm{d}}$ the downstream transit time).

$$
\begin{gathered}
t_{u}=L /(c+u \cdot \cos \varphi) \\
t_{d}=L /(c-u \cdot \cos \varphi) \\
u=(L / 2 \cos \varphi) \cdot\left(\mathrm{t}_{\mathrm{u}}-\mathrm{t}_{\mathrm{d}}\right) /\left(\mathrm{t}_{\mathrm{u}} \cdot \mathrm{t}_{\mathrm{d}}\right) \\
\text { flow }=\pi \cdot \mathrm{r}^{2} \cdot \mathrm{u}
\end{gathered}
$$

where $L$ is the length of the sound path, $c$ is the speed of sound, $u$ is the mean gas flow velocity on the sound transmission path, and $\phi$ is the angle $\left(40^{\circ}\right)$ between the flow channel and the sound path. The elimination of $c$ by solving the transit time equations minimizes the effect of gas density on the estimate of flow.

The fraction of the tracer gas $\mathrm{SF}_{6}$ is measured using the molar mass. The molar mass of the gas mixture is dependent on gas composition, temperature, humidity, and pressure. Tem- 
perature differences between inspiratory and expiratory gas are corrected using a temperature model. In the present study we have not examined the validity of the temperature model in a subject spontaneously breathing in room air (when changes in temperature and humidification are more pronounced than in a subject ventilated with humidified and heated gas). An increase in pressure during CMV leads to underestimation of the absolute value of the molar mass as shown in Figure 2. However, the $\mathrm{SF}_{6}$ fraction is measured using the deltaMM compared with baseline values before the $\mathrm{SF}_{6}$ washin, and we have demonstrated that the accuracy of FRC measurements was not affected by airway pressure.

FRC can be measured routinely in children and infants by using a nitrogen washout, and the open-circuit technique proposed by Sivan et al. $(4,5)$ shows high accuracy and reproducibility. However, this nitrogen washout technique needs bulky equipment, two ventilators, a mass spectrometer, and a relatively complex setup and calibration. In addition FRC cannot be measured by nitrogen washout in patients who require $>70-80 \%$ oxygen. For these reasons it is unsuitable for clinical purposes. More recently $\mathrm{SF}_{6}$ washout techniques were described using an infrared $\mathrm{SF}_{6}$ analyzer $(9,12-14,23)$. The signal-to-noise ratio of the used infrared analyzer is very low and could therefore not be used during $\mathrm{SF}_{6}$ washin. The infrared $\mathrm{SF}_{6}$ analyzer has a nonlinear response to $\mathrm{SF}_{6}$ changes, which makes calibration difficult. The study by Vilstrup et al. (23) showed in a mechanical lung model a similar accuracy and reproducibility (mean coefficient of variation, $4 \%$ ) of the infrared analyzer method compared with our study. In the same study, in vivo measurements in rabbits, however, showed a higher coefficient of variation (mean, $8 \%$; range, $0-17 \%$; our study, mean, $5.5 \%$; range, $0-15.3 \%$ ). Unfortunately the analyzer used by Vilstrup et al. (23) is no longer available and direct comparison was impossible. Despite the high purity of $\mathrm{SF}_{6}$ used in this study (99.99\%), there is a very small chance that the $\mathrm{SF}_{6}$ gas is contaminated with toxic $\mathrm{SF}_{5}$. As the concentration of $\mathrm{SF}_{6}$ used was $2-4 \%$, the concentration of any contaminant was $<0.0025 \%$.

The MBNW in ventilated infants and children first described by Sivan et al. (4) measures only FRC without giving additional information about ventilation distribution. Analysis of the washout curve is only possible when end-expiratory $\mathrm{SF}_{6}$ concentration can be measured breath by breath. We showed in normal animals and in children with lung disease that ventilation distribution improves with increasing CPAP or PEEP. However, these findings are based on small numbers only and need to be confirmed in a larger group of healthy children. Vilstrup et al.(23) used the pulmonary clearance delay to measure homogeneity of ventilation but could not demonstrate in small neonates an improvement of ventilation distribution with higher PEEP. Larsson et al.(24) have previously shown that LCI, moment ratio analysis, and pulmonary clearance delay are affected by changes in tidal volume and series dead space. Therefore, Habib and Lutchen(16) introduced the AMDN, which uses the cumulative expired alveolar volume for moment ratio analysis. Habib and Lutchen(16) showed that AMDN is superior to conventional moment ratio analysis(10) and less sensitive to breathing pattern. In our small and very heterogeneous group of investigated children, AMDN appeared to improve (more homogeneous ventilation) as PEEPincreased, but the change did not reach statistical significance. The aim of this pilot study was not to characterize different lung pathologies but only to demonstrate the application of the new technique in a clinical situation. The bed side measurement of ventilation distribution is a potentially valuable research tool for investigation of pathophysiology and treatment of lung disease. It is also likely to be clinically useful for the titration of drug treatment (e.g. application of surfactant in acute respiratory distress syndrome), physiotherapy, or mechanical ventilation in children with lung disease.

Limits of the method. Leaks around the ETT are very common in infants and may cause errors when FRC is measured. Leaks are not always present and, when they are present, may be reduced by applying pressure over the trachea. During mechanical ventilation, leaks are usually greatest during inspiration, whereas the washin or washout volume of $\mathrm{SF}_{6}$ is calculated from expiratory signals. At least in theory, the $\mathrm{SF}_{6}$ washin and washout method should therefore be less sensitive to leaks than, for example, helium equilibration (7). $\mathrm{SF}_{6}$ washout shares the limitations of other gas washout techniques (17), which measure only the volume of gas that communicates with the airway opening and cannot measure gas trapped within the lung.

The empirical application of PEEP in diseased lungs is usually based only on clinical judgment. As many other studies have shown $(4,12,13)$, at higher levels of PEEP, the FRC increases, areas of collapsed lung are reopened, and some of the trapped air may be recruited. This normally leads to an improvement in oxygenation. In our study, we have given some examples that ventilation distribution (AMDN1, AMDN2) improves with increasing PEEP using an animal model. Theoretically, any increase in FRC without improvement in moment ratios should be avoided to prevent lung damage caused by positive-pressure ventilation. However, further studies to compare the relationship between FRC, moment ratios, gas exchange, and type of lung injury are needed to prove this hypothesis.

Conclusion. We present a technique of measuring FRC in spontaneously breathing or mechanically ventilated patients based on $\mathrm{SF}_{6}$ washin and washout. A unique feature of the technique is a compact all-in-one sensor, which measures flow, volume, and gas concentration with the same ultrasonic sound signal. FRC measurement with this technique is highly accurate and reproducible and also gives an estimate of ventilation inhomogeneity in healthy or diseased lungs. Unlike the nitrogen washout technique, it is easy to use for routine bedside testing as it does not require complex bulky equipment and can be used in patients who require $80-100 \%$ inspired oxygen. Further studies in animals and larger groups of children are needed to compare its accuracy with that of plethysmography and nitrogen washout.

\section{REFERENCES}

1. Darling RC, Richards DW, Courand A 1940 Studies on intrapulmonary mixture of gas: open circuit method for measuring residual air. J Clin Invest 19:609-618 
2. Saidel GM, Salmon RB, Chester EH 1975 Moment analysis of multibreath lung washout. J Appl Physiol 38:328-334

3. Gerhardt T, Hehre D, Bacalari E, Watson H 1985 A simple method for measuring functional residual capacity by $\mathrm{N}_{2}$ washout in small animals and newborn infants. Pediatr Res 19:1165-1169

4. Sivan Y, Deakers TW, Newth CJL 1990 Functional residual capacity in ventilated infants and children. Pediatr Res 28:451-454

5. Sivan Y, Deakers TW, Newth CJL 1990 An automated bedside method for measuring functional residual capacity by $\mathrm{N}_{2}$ washout in mechanically ventilated children. Pediatr Res 28:446-450

6. Wrigge H, Sydow M, Zinserling J, Neumann P, Hinz J, Buchardi H 1998 Determination of functional residual capacity (FRC) by multibreath nitrogen washout in a lung model and in mechanically ventilated patients. Intensive Care Med 24:487-493

7. Schaanning CG, Gulsvik A 1973 Accuracy and precision of helium dilution technique and body plethysmography in measuring lung volumes. J Clin Lab Invest 32:271-277

8. Stokke T, Hensel I, Buchardi H 1985 A simple method for determination for the functional residual capacity during artificial ventilation. Anaesthetist 30:124-129

9. Jonmarker C, Jansson L, Jonson B, Larsson A, Werner O 1985 Measurement of functional residual capacity by sulfur hexafluoride washout. Anesthesiology 63:89-95

10. Saidel GM, Saniie J, Chester EH 1978 Modeling and moments of multibreath lung washout. Ann Biomed Eng 6:126-137

11. Saidel GM, Saniie J, Chester EH 1980 Lung washout during spontaneous breathing: parameter estimation with a time-varying model. Comput Biomed Res $13: 446-457$

12. Schulze A, Schaller P, Töpfer A, Kirplani H 1994 Measurement of functional residual capacity by sulfur hexafluoride in small-volume lungs during spontaneous breathing and mechanical ventilation. Pediatr Res 35:494-499
13. Vilstrup CT, Björkulund J, Larsson A, Lachmann B, Werner O 1992 Functional residual capacity and ventilation inhomogeneity in mechanically ventilated small neonates. J Appl Physiol 73:276-283

14. East TD, Wortelboer JM, van Ark E, Bloem FH, Peng L, Pace NL, Crapo RO, Drews D, Clemmer TP 1990 Automated sulfur hexafluoride washout functional residual capacity measurement system for any mode of mechanical ventilation as well as spontaneous respiration. Crit Care Med 18:84-91

15. Buess C, Pietsch P, Guggenbühl W, Koller EA 1991 A pulsed diagonal-beam ultrasonic airflow meter. J Appl Physiol 61:1195-1199

16. Habib RH, Lutchen KR 1991 Moment analysis of multiple nitrogen washout based on an alveolar gas dilution number. Am Rev Respir Dis 144:513-519

17. Schibler A, Schneider M, Frey U, Kraemer R 2000 Moment ratio analysis of multiple breath nitrogen washout in infants with lung disease Eur Respir J 15:1094-1101

18. Sivan Y, Hammer J, Newth CJL 1994 Measurement of high volumes by nitrogen washout method. J Appl Physiol 77:1562-1564

19. Altman DG, Bland JM 1983 Measurement in medicine: the analysis of method comparison studies. Statistician 32:307-317

20. Brunner JX, Wolff G, Cumming G, Langenstein H 1985 Accurate measurement of $\mathrm{N}_{2}$ volumes during $\mathrm{N}_{2}$ washout requires dynamic adjustment of delay time J Appl Physiol 59:1008-1012

21. Grenvik A, Hedstrand U, Sjögren H 1966 Problems in pneumotachography. Acta Anaesthesiol Scand 10:147-155

22. Knox WJ, Mills CJ, Hale T 1991 Correction of pneumotachograph signal for changes in viscosity during nitrogen washout Clin Phys Physiol Meas 12:359-365

23. Vilstrup CT, Björkulund LJ, Werner O, Larsson A 1996 Lung volumes and pressure volume relations of the respiratory system in small ventilated neonates with severe respiratory distress syndrome. Pediatr Res 39:127-133

24. Larsson A, Jonmarker C, Werner O 1988 Ventilation inhomogeneity during controlled ventilation: which index should be used? J Appl Physiol 65:2030-2039 